\title{
Multi-level evidence that circulating CKI 8 is a biomarker of tumour burden in colorectal cancer
}

\author{
A Greystoke', , E Dean 1,6, MP Saunders², J Cummings', A Hughes ${ }^{3}$, M Ranson', C Dive' and AG Renehan ${ }^{*, 1,4,5}$ \\ 'Clinical and Experimental Pharmacology (CEP) Group, Paterson Institute for Cancer Research, University of Manchester, Manchester, UK; ' ${ }^{2}$ Department \\ of Clinical Oncology, The Christie NHS Foundation Trust, Manchester, UK; ${ }^{3}$ Astra Zeneca, Alderley Park, Macclesfield, UK; ${ }^{4}$ Department of Surgery, \\ Manchester Academic Health Science Centre, The Christie NHS Foundation Trust, University of Manchester, Wilmslow Road, Manchester M20 4BX, UK; \\ ${ }^{5}$ Institute of Cancer Sciences, University of Manchester, Manchester, UK
}

BACKGROUND: Circulating total cytokeratin 18 (tCKI8) and/or caspase cleaved cytokeratin I8 (cCKI8) (measured by M65 and M30 enzyme-linked immunosorbent assays (ELISAs), respectively) are used as pharmacodynamic (PD) biomarkers of epithelial cell death in clinical trials. Having validated these ELISAs, we assessed their utility in colorectal cancer (CRC).

METHODS: We applied the assays in several settings: 53 controls; 97 patients undergoing surgery and 74 patients with metastatic CRC undergoing chemotherapy (55 first line; 56 patients with repeated sampling through chemotherapy). Prognostic significance was evaluated using Kaplan-Meier life tables and Cox models; PD utility was assessed by analysis of repeated measures.

RESULTS: Median CCKI 8 and tCKI 8 levels were elevated in patients with cancer (both $P=0.000 \mathrm{I}$ ), and among cancer patients, there were increasing trends from early to advanced stages (both $P_{\text {trends }}=0.000 \mathrm{I}$ ). Increasing $\mathrm{tCK} \mid 8$ predicted for reduced survival after surgery with curative intent (adjusted hazard ratio (HR) for doubling in concentration 1.77, $95 \%$ Cl: 1.04, 3.0I) and after first-line chemotherapy in metastatic disease (adjusted HR per doubling in concentration $=1.78,95 \% \mathrm{Cl}$ : I.37, 2.30). In patients with progressive disease during chemotherapy, repeated sampling revealed profiles with high baselines and progressive upwardly increases after cycle I.

CONCLUSION: This study provides evidence for cytokeratin $18(C K I 8)$ as a prognostic and PD biomarker in patients with CRC and supports continued deployment of circulating CKI 8 in biomarker-enhanced trials.

British Journal of Cancer (2012) 107, 1518-1524. doi:I0.1038/bjc.2012.416 www.bjcancer.com

Published online 20 September 2012

(c) 2012 Cancer Research UK

Keywords: apoptosis; biomarkers; colorectal cancer; tumour burden; prognosis

Colorectal cancer (CRC) is the second most common cancer in Europe accounting for 183000 new cancers per year. The integration of mechanism-based therapies (MBTs, e.g., cetuximab or bevacizumab) with chemotherapy in CRC has expanded treatment options, resulting in survival gains in some patients (Cunningham et al, 2010). With the introduction of MBTs, there is a need to develop biomarkers to prioritise new agent development (predictive and prognostic biomarkers), optimise dosing schedules (pharmacodynamic (PD) biomarkers), and rationalise combination selection (Cummings et al, 2010).

Circulating products of epithelial cell death have been used as PD biomarkers for MBT responses both in animal models (Olofsson et al, 2009) and in clinical trials (Cummings et al, 2008). The M30 apoptosense and M65 enzyme-linked immunosorbent assays (ELISAs) detect circulating cytokeratin 18 (CK18) fragments: the M30 ELISA selectively detects caspase cleaved CK18 (cCK18) (apoptosis), whereas the M65 assay detects cCK18 and uncleaved CK18 (total cell death, e.g., apoptosis and necrosis) (Cummings et al, 2005, 2006). We previously validated these assays (Cummings et al, 2005, 2006) and determined they are 'fit for purpose' (Greystoke et al, 2008). To-date, we have reported their

*Correspondence: Dr AG Renehan; E-mail: arenehan@picr.man.ac.uk

${ }^{6}$ These authors contributed equally to this work.

Received 30 March 2012; revised 21 August 2012; accepted 22 August 2012; published online 20 September 2012 potential utility in testicular cancer (de Haas et al, 2008), pancreatic cancer (Dive et al, 2010), small cell lung cancer (SCLC) (Hou et al, 2009; Dean et al, 2011), and lymphoma (Greystoke et al, 2011), and evaluated their performance in early clinical trials (Dean et al, 2009; Gandhi et al, 2011). A few studies with low sample numbers have evaluated serum cCK18 and total CK18 (tCK18) in patients with gastrointestinal cancers (Scott et al, 2009) and CRC (Ausch et al, 2009a, b).

Previous studies in SCLC and testicular cancer suggested that elevated baseline tCK18 levels are associated with poor prognosis, and that in these chemo-sensitive tumours, response is accompanied by falling tCK18 levels (de Haas et al, 2008; Hou et al, 2009). In a colorectal xenograft model, tCK18 correlated with tumour burden, whereas measurement of cCK18 correlated with treatment-related tumour death (Cummings et al, 2008). Based on these observations, we hypothesised that (i) elevated levels of tCK18 at baseline are associated with poor prognosis, (ii) circulating cCK18 levels are associated with tumour apoptosis following therapy, and (iii) circulating tCK18 correlates with tumour burden in CRC.

\section{PATIENTS AND METHODS}

\section{Study design and patients}

We used a mixed cross-sectional prospective design to assess the utility of serum cCK18 and tCK18 across multiple clinical settings 


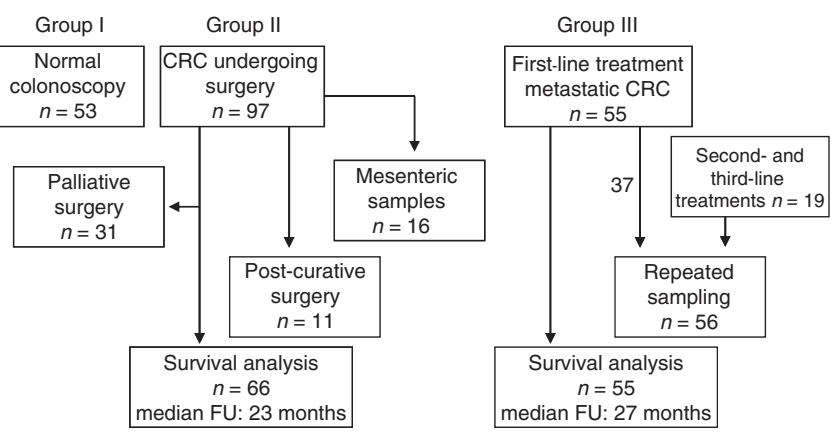

Figure I Flow diagram of analyses - a mixed cross-sectional prospective design. Abbreviations: $\mathrm{CRC}=$ colorectal cancer; $\mathrm{FU}=$ follow-up.

in 224 subjects (Figure 1): (i) 53 individuals undergoing screening colonoscopy (controls); (ii) 97 undergoing surgery (Renehan et al, 2000); (iii) 74 patients undergoing palliative chemotherapy. Survival analysis was restricted to 66 surgery patients who underwent curative resection and 55 patients undergoing first-line chemotherapy. In addition, 56 patients underwent repeated sampling throughout chemotherapy.

All treatments were carried out at the Christie NHS Foundation Trust, Manchester, UK. The study was carried out according to the Good Clinical Practice guidelines, and following local ethics committee approval.

\section{Sampling and assays}

Blood was collected in tubes containing a silica clot activator and centrifuged at $2000 \mathrm{~g}$ for $10 \mathrm{~min}$ to obtain serum. The sample collection, processing, storage, and measurement of cCK18 and tCK18 were as previously described (Greystoke et al, 2008) (see Supplementary Material).

Baseline samples were collected $30 \mathrm{~min}$ prior to colonoscopy (group I), the day before surgery (group II), or the first day of chemotherapy (group III). Venous mesenteric sampling was performed intra-operatively prior to ligation of the pedicle drawing blood from the main draining vessel, for example, the inferior mesenteric vein for recto-sigmoid tumours. Repeated sampling in group III patients varied depending on clinical practice, but were typically collected on days $1,3,8,15,21,28,35$, 42,49 , and 56 , and thereafter determined by clinical indication.

\section{Statistical analysis}

CK18 biomarker distributions in patients with CRC were highly skewed (see Supplementary Table 1). Thus, comparisons were performed using Mann-Whitney $U$ - and Kruskal-Wallis tests; paired samples were compared using Wilcoxan rank tests; and nonparametric tests for trend across ordered groups (Cuzick, 1985).

For prognostic analyses, we followed the REMARK guidelines (McShane et al, 2005). Survival curves were derived using KaplanMeier analyses and comparisons performed using log-rank tests. Cox models were applied to assess whether cCK18 and tCK18 were independent prognostic factors for survival. We used a seven-step approach advocated by Hosmer and Lemeshow (2000), forcing cCK18 and tCK18 data separately into the final model. Biomarkers were handled as both continuous and categorical variables in separate models - the former, to test for independent prognostic significance; the latter, to allow extension of the univariate survival analyses and explore for potential clinically relevant cut points. For the continuous data, to reduce the right skewness of the distributions of the CK18 concentrations, the base 2 logarithmic transformation was used, which leads to a convenient interpretation: the hazard ratio (HR) associated with a change of one unit on the $\log 2$ scale corresponds to the HR associated with a doubling in CK18 level on the original scale. For all models, we excluded variables if the partial likelihood test $P$-value $>0.05$. In initial models for surgically treated patients, we considered age, sex, stage, and carcinoembryonic antigen (CEA: as tertiles); in initial models for patients with metastatic disease, we considered age, sex, presence of liver metastases, treatment regimens, CEA, alkaline phosphatise, aspartate transaminase, and lactate dehydrogenase as continuous variables (after natural log transformation). As these analytes correlated with each other and with cCK18 and tCK18 (see Supplementary Table 2), models were tested for co-linearity. Predictive accuracy and calibration were tested, respectively, using a concordance index (C-statistic; values ranging from 0.5 to 1.0 ) and the calibration statistic, analogous to the Hosmer-Lemeshow goodness-of-fit test (with values $\leqslant 20$ indicating good agreement).

In patients undergoing chemotherapy, we assessed PD response using analyses of repeated measures but for pragmatic purposes, we limited analyses to regression - joint mixed effects modelling may better test for differences between biomarkers, but the complexity of such modelling was beyond the scope of this paper (Steele, 2008). Instead, each patient was handled as a dummy variable to take account of intra-individual correlation. We investigated potential nonlinear relationships between the biomarkers and time on treatment using restricted cubic splines with three knots at 3, 14, and 21 days, chosen to represent immediate changes, and two subsequent time points at which future biomarker-directed trials may inform early decisions on continuation of trial drug. Carcinoembryonic antigen was checked at the start of every cycle - a $20 \%$ rise after cycle 2 was considered clinically relevant, while a rise in tCK18 $>12 \%$ from cycle 2 onwards was considered a positive signal; larger than the documented intra-person variability in the assay (Cummings et al, 2006). Response to therapy was assessed by CT scan every 2 months during therapy. Patients were categorised into progressive disease or clinical benefit (either stable disease or partial remission) based on RECIST 1.0 (Therasse et al, 2000). All analyses were performed using STATA version 11.1 (College Station, TX, USA).

\section{RESULTS}

\section{Baseline characteristics}

Baseline characteristics are shown in Table 1. The proportion of men was greater in subjects receiving chemotherapy $(78.2 \%)$ compared with controls (54.7\%) and surgically treated subjects (51.6\%; $P=0.004)$; patients with cancer were older $(P=0.0004)$ and had a lower BMI $(P=0.02)$ than controls. However, in the control group serum, cCK18 and tCK18 were uninfluenced by gender, age, and BMI (see Supplementary Tables 3-5). As might be expected, median CEA levels were elevated in patients receiving palliative chemotherapy compared with surgically treated patients $(P=0.0002)$.

\section{Comparisons of cancer patients $v s$ controls}

Concentrations were significantly increased in patients with cancer for tCK18 (median (inter-quartile range): 410 (287, 942) vs 185 $\left.(151,235) \mathrm{Ul}^{-1}, P=0.0001\right)$ and $\operatorname{cCK} 18(162(106,297)$ vs 113 $\left.(168,89) \mathrm{Ul}^{-1}, P=0.0001\right)$ compared with those in controls (see Supplementary Table 6). Among patients with cancers, there were trends of increasing median concentrations from stage I and II (surgery), through stage III and locally advanced (surgery), to patients with metastatic disease for both biomarkers (both $P_{\text {trends }}=0.0001$ ) (Figure 2).

Among 16 patients undergoing resection, median levels from tumour-draining mesenteric veins were higher than those in peripheral blood for tCK18 (medians: 446 vs $308 \mathrm{Ul}^{-1}$, 
Table I Baseline characteristics for groups I, II, and III

\begin{tabular}{|c|c|c|c|}
\hline & $\begin{array}{c}\text { Group I } \\
\text { Normal } \\
\text { colonoscopy }\end{array}$ & $\begin{array}{c}\text { Group II } \\
\text { CRC } \\
\text { undergoing } \\
\text { surgery }\end{array}$ & $\begin{array}{c}\text { Group III } \\
\text { First-line } \\
\text { metastatic } \\
\text { CRC }\end{array}$ \\
\hline $\begin{array}{l}\text { No. of patients } \\
\text { Men:women } \\
\text { Median age (IQR) years }\end{array}$ & $\begin{array}{c}53 \\
29: 24 \\
60.2 \\
(58.0-62.8)\end{array}$ & $\begin{array}{c}97 \\
50: 47 \\
68.1 \\
(55.3-75.3)\end{array}$ & $\begin{array}{c}55 \\
43: 12 \\
65.0 \\
(57.0-72.0)\end{array}$ \\
\hline $\begin{array}{l}\text { Performance status } \\
0(\%) \\
1(\%) \\
2(\%)\end{array}$ & $\begin{array}{l}\text { NA } \\
\text { NA } \\
\text { NA }\end{array}$ & $\begin{array}{l}65(67) \\
20(21) \\
12(12)\end{array}$ & $\begin{array}{l}25(45) \\
20(36) \\
10(18)\end{array}$ \\
\hline $\begin{array}{l}\text { Tumour stage }^{\mathrm{a}} \\
\text { I and II (\%) } \\
\text { III and IV (\%) } \\
\text { Distant metastases - no liver (\%) } \\
\text { Distant metastases - liver (\%) }\end{array}$ & $\begin{array}{l}\text { NA } \\
\text { NA } \\
\text { NA } \\
\text { NA }\end{array}$ & $\begin{array}{c}53(55) \\
44(45) \\
\text { NA } \\
\text { NA }\end{array}$ & $\begin{array}{c}\text { NA } \\
\text { NA } \\
21(38) \\
34(62)\end{array}$ \\
\hline $\begin{array}{l}\text { Surgical treatment } \\
\text { Curative segmental resection (\%) } \\
\text { Curative - exenterative surgery (\%) } \\
\text { Curative segmental resection with } \\
\text { metastases (\%) } \\
\text { Palliative (\%) }\end{array}$ & $\begin{array}{l}\text { NA } \\
\text { NA } \\
\text { NA } \\
\text { NA }\end{array}$ & $\begin{aligned} & 50(52)^{b} \\
& 4(4)^{c} \\
& 12(12)^{d} \\
& 31(32)\end{aligned}$ & $\begin{array}{l}\text { NA } \\
\text { NA } \\
\text { NA } \\
\text { NA }\end{array}$ \\
\hline $\begin{array}{l}\text { Chemotherapy } \\
\text { Ox+ (5-FU/Cap) (\%) } \\
\text { Ir + (5-FU/Cap) (\%) } \\
\text { Cap (\%) } \\
\text { Others (\%) }\end{array}$ & $\begin{array}{l}\text { NA } \\
\text { NA } \\
\text { NA } \\
\text { NA }\end{array}$ & $\begin{array}{l}\text { NA } \\
\text { NA } \\
\text { NA } \\
\text { NA }\end{array}$ & $\begin{aligned} & 28(51) \\
& 6(11) \\
& 11(20) \\
& 10(18)\end{aligned}$ \\
\hline Median serum CEA (IQR) $\mathrm{ng} \mathrm{ml}^{-1}$ & NA & $7(3-5 \mid)$ & $18.5(3-95)$ \\
\hline
\end{tabular}

Abbreviations: $\mathrm{Cap}=$ capecitabine; $\mathrm{CEA}=$ carcinoembryonic antigen; $\mathrm{CRC}=$ colorectal cancer; $\mathrm{FU}=$ fluorouracil; $\mathrm{Ir}=$ irinotecan; $\mathrm{IQR}=$ inter-quartile range; $\mathrm{NA}=$

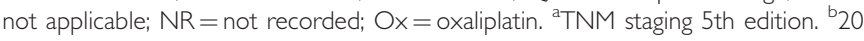
anterior resections, 10 abdomino-perineal resections, 2 Hartmann resections, 7 left hemi-colectomies, 6 right hemi-colectomies, 3 subtotal colectomies, and 2 local excisions. 'Three total pelvic clearances and one cytoreductive surgery. ${ }^{\mathrm{d}}$ Three anterior resections; one abdomino-perineal resection; one Hartmann resection; four left hemi-colectomies; two right hemi-colectomies, and one total pelvic clearance.

$\left.P_{\text {paired }}=0.003\right)$ and to a lesser extent cCK18 (189 vs $98 \mathrm{Ul}^{-1}$, $\left.P_{\text {paired }}=0.03\right)$ (see Supplementary Figure 1A). Median levels of tCK18 reduced after surgery in 11 patients undergoing resection (medians: 310 vs $\left.268 \mathrm{Ul}^{-1}, P=0.07\right)$, but not cCK18 $(P=0.37)$ (see Supplementary Figure 1B).

\section{Prognostic modelling}

In 66 patients who underwent curative surgery, increasing tertiles of tCK18 and cCK18 were associated with significantly reduced cancer-specific survival (log rank: $P=0.0284$ and $P=0.0097$, respectively) (Figure $3 \mathrm{~A}$ ). For the 55 patients receiving palliative first-line chemotherapy, increasing biomarker levels were associated with reduced overall survival for tCK18 $(P=0.0017)$, but less so for cCK18 $(P=0.0690)$ (Figure 3B).

We tested these associations further using multivariate Cox proportional hazards models. In surgically treated patients, the models with tCK18 and cCK18 as continuous variables (log transformed to base 2) were borderline significant, but in general, the prognostic associations of tCK18 and cCK18 were attenuated after adjustment included stage (Table 2). However, in patients with metastatic CRC, increasing concentrations of the biomarkers were independently significant for tCK18 (HR per doubling in concentration $=1.78,95 \% \mathrm{CI}: 1.37,2.30, P<0.001)$ and $\mathrm{cCK} 18(\mathrm{HR}$
A
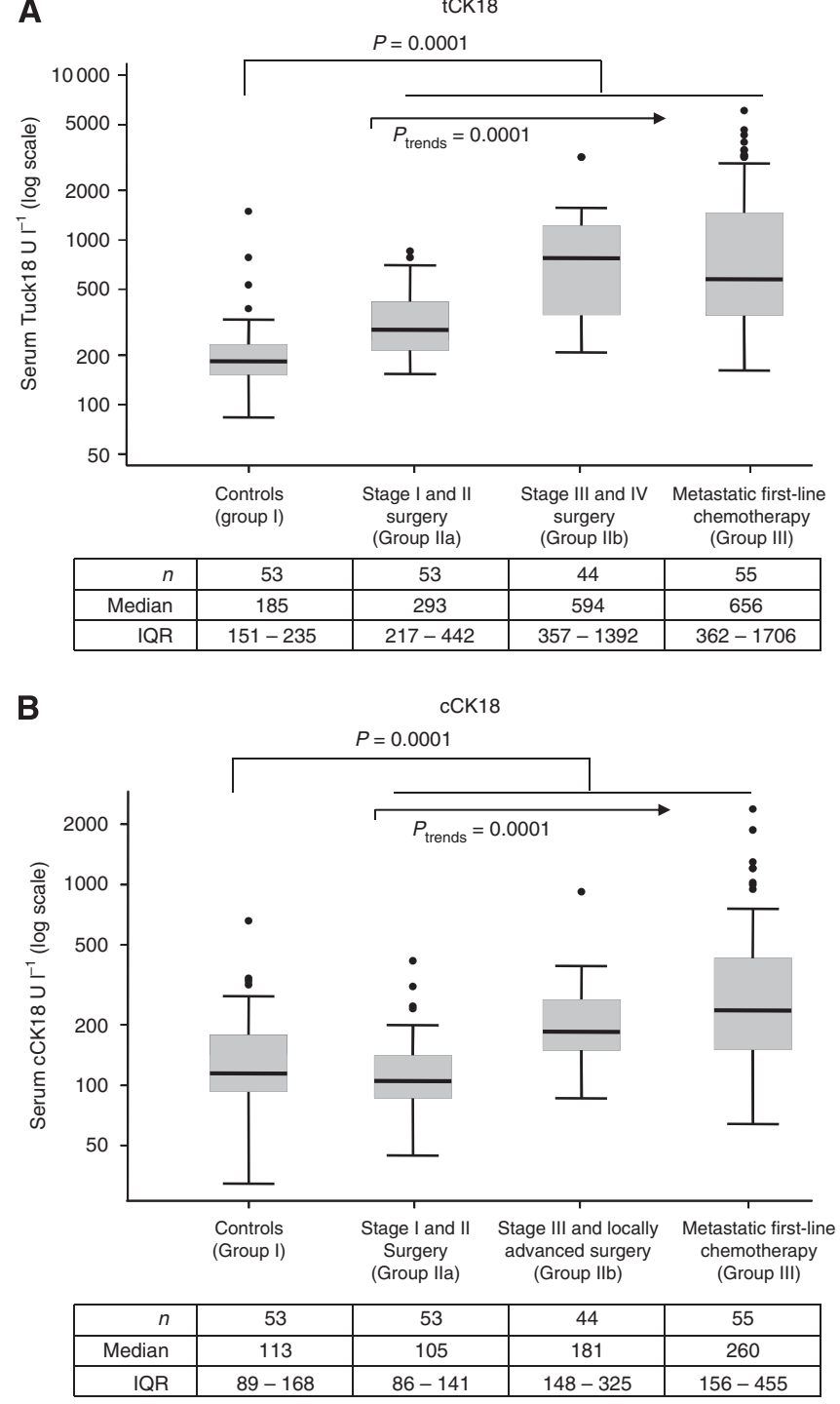

Figure 2 (A) Serum tCKI8 concentrations by disease groups of increasing stage. (B) Serum CCKI8 concentrations by disease groups of increasing stage. The $P$-values at the top of each figure represent comparisons of medians for all patients with cancer vs controls. The tests for trends used the Cuzick test for nonparametric distributions of ordered groups. Pairwise comparisons (Kruskal-Wallis tests) were also performed as follows: for tCK I 8, group lla vs I, $P=0.000 \mathrm{I}$; llb vs Ila, $P=0.000 \mathrm{I}$; III vs Ilb, $P=0.486$; and for cCKI8, Ila vs I, $P=0.615$; Ilb vs Ila, $P=0.000 \mathrm{I}$; III vs Ilb, $P=0.084$. Note: $\log$ scale on y axis. Abbreviation: $I Q R=$ inter-quartile range.

per doubling in concentration $=1.93,95 \% \mathrm{CI}: 1.32,2.81, P=0.001)$ (Table 3).

\section{Longitudinal chemotherapy-related effects}

Fifty-six patients receiving chemotherapy underwent repeated sampling (37 patients first-line and 19 patients second- or thirdline chemotherapy). Patients with disease progression on chemotherapy had higher median baseline tCK18 (999 vs $389 \mathrm{Ul}^{-1}$, $P=0.004)$ and cCK18 (383 vs $\left.225 \mathrm{Ul}^{-1}, P=0.019\right)$ levels than patients experiencing clinical benefit. In 24 patients with progressive disease, there was an initial decrease in biomarker levels during cycle 1 followed by a progressive upward trend thereafter (Figure 4). In 32 patients with clinical benefit, an initial decrease in biomarker was observed in tCK18 only with no subsequent change. 
A

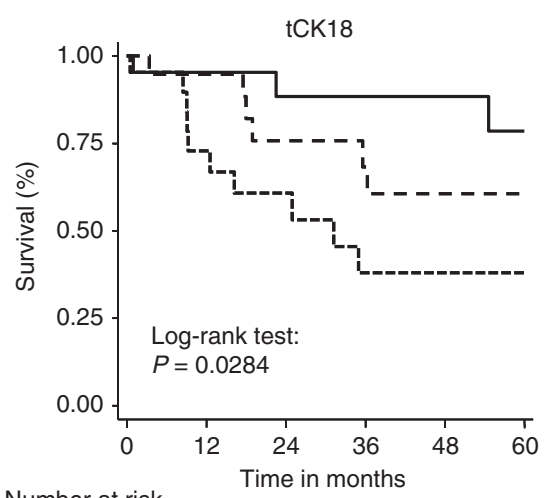

Cancer-specific survival - surgically treated

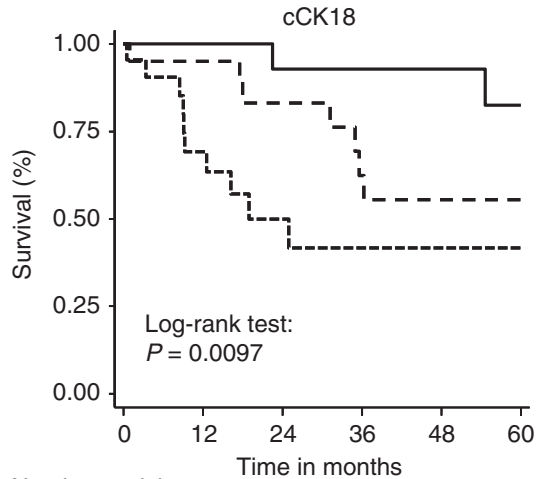

Number at risk

$\begin{array}{lccccc}22 & 15 & 12 & 12 & 11 & 8 \\ 22 & 17 & 13 & 9 & 7 & 7 \\ 22 & 12 & 6 & 5 & 5 & 5\end{array}$

B

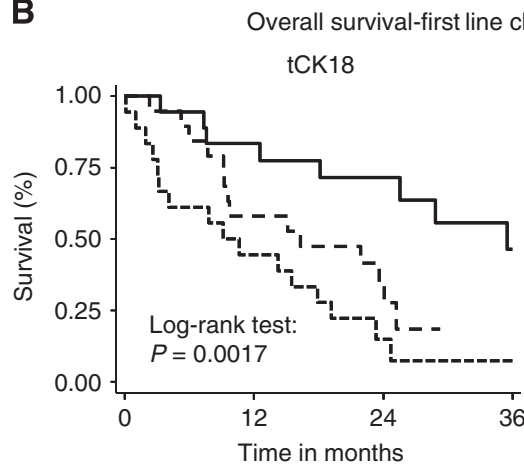

Number at risk

Tertile 118

Tertile 219

Tertile 318

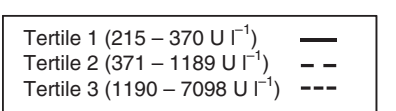

Tertile $1\left(45-95 \mathrm{UI}^{-1}\right)$

Tertile $2\left(96-148 \cup 1^{-1}\right)$

Tertile $3\left(149-920 \cup^{-1}\right) \quad--$

Figure 3 (A) Cancer-specific survival in patients with CRC undergoing surgical resection with curative intent. (B) Overall survival in patients undergoing first-line conventional chemotherapy for metastatic CRC.

We noted examples where CK18 was more informative than CEA. Thus, of 24 patients with progressive disease on therapy, 9 patients had clinically relevant increases in CEA prior to tCK18 (median 21 days; range 7-28), 3 patients had simultaneous increases in CEA and tCK18, 5 patients had increases in tCK18 prior to CEA (median 21 days; range 7-48), and 7 patients had increases in tCK18 (at median 49 days post start of therapy; range 21-147), but never had clinically relevant CEA rises. Additionally of the 74 patients with metastatic disease who received chemotherapy, 21 subjects had undetectable CEA or levels at the lower limit of quantification.

Finally, we explored whether changes in tCK18 and cCK18 reflect treatment-related toxicity. Using aspirate transaminase levels as a surrogate of hepatic dysfunction, we found no patients with protracted elevated serum concentrations (see Supplementary Figure 2).

\section{DISCUSSION}

This study assessed the prognostic and PD utility of circulating CK18 in a range of clinical settings in CRC. The study demonstrated that serum tCK18 and cCK18 levels were elevated in patients with cancer compared with controls, and among patients with cancers, there were trends of increasing median concentrations from early to advanced stages. Levels were higher in tumour-draining venous samples compared with peripheral sampling; and levels fall following tumour resection. Collectively, these observations support the hypothesis that these biomarkers are tumour-derived.

The utility of tCK18 and cCK18 as a diagnostic biomarker of malignancy is limited by the overlap with healthy controls. However, elevated levels of tCK18 and cCK18 prior to treatment were associated with poorer prognosis in both the surgical and metastatic setting, though in the surgical setting much of this prognostic impact was accounted for by associations with stage.

We explored the utility of serum tCK 18 and $\mathrm{cCK} 18$ as a PD biomarker in patients receiving chemotherapy for metastatic CRC and found that patients with progressive disease on therapy have elevated serum CK18 pre-treatment, tend to show a reduction in these biomarkers at 14-21 days, but then levels progressively increase, returning to and exceeding pre-treatment levels. This is 
Table 2 Multivariate Cox models of total CKI8 (tCKI8) and caspase cleaved CKI8 (cCKI8) prognostic influence on cancer-specific survival in patients with colorectal cancer undergoing surgical resection $(n=66)$

\begin{tabular}{|c|c|c|c|c|}
\hline & Categories $\left(\mathrm{UI}^{-1}\right)$ & Hazard ratio $(95 \% \mathrm{Cls})$ & $P_{\text {continuous }}$ & $P_{\text {vs referent }}$ \\
\hline \multicolumn{5}{|l|}{ tCKI8 model ${ }^{a}$} \\
\hline Continuous ${ }^{b}$ & Per unit log2 & $1.77(1.04-3.01)$ & 0.036 & \\
\hline Tertile $2(n=22)$ & $243-430$ & $1.47(0.34-6.31)$ & & 0.601 \\
\hline Tertile $3(n=22)$ & $431-3608$ & $2.82(0.72-11.07)$ & & 0.136 \\
\hline \multicolumn{5}{|l|}{ cCK 18 model $\left.\right|^{\mathrm{a}}$} \\
\hline \multicolumn{5}{|l|}{ Categorical } \\
\hline Tertile I $(n=22)$ & $45-95$ & 1.00 & & \\
\hline Tertile $2(n=22)$ & $96-148$ & $3.41(0.69-17.01)$ & & 0.134 \\
\hline Tertile $3(n=22)$ & |49-920 & $4.72(0.94-23.75)$ & & 0.060 \\
\hline
\end{tabular}

Abbreviation: $\mathrm{Cl}=$ confidence interval. The calibration and performance characteristics for cancer-specific survival model for M65 were: Harrell's C-statistic $=0.5557$ and likelihood ratio for goodness-of-fit $=11$. 924 ; for $\mathrm{M} 30, \mathrm{C}=0.7684$ and likelihood ratio for goodness-of-fit $=13.946$. ${ }^{\mathrm{a}} \mathrm{All}$ final models included were adjustment for age, sex, stage, and tCKI8 and CCKI8, respectively. ${ }^{b}$ Interpreted as change in hazard ratio associated with a doubling in CKI8 level on the original scale.

Table 3 Multivariate Cox models of total CKI8 (tCKI8) and caspase cleaved CKI8 (cCKI8) prognostic influence on overall survival in patients undergoing first-line conventional chemotherapy for metastatic colorectal cancer $(n=55)$

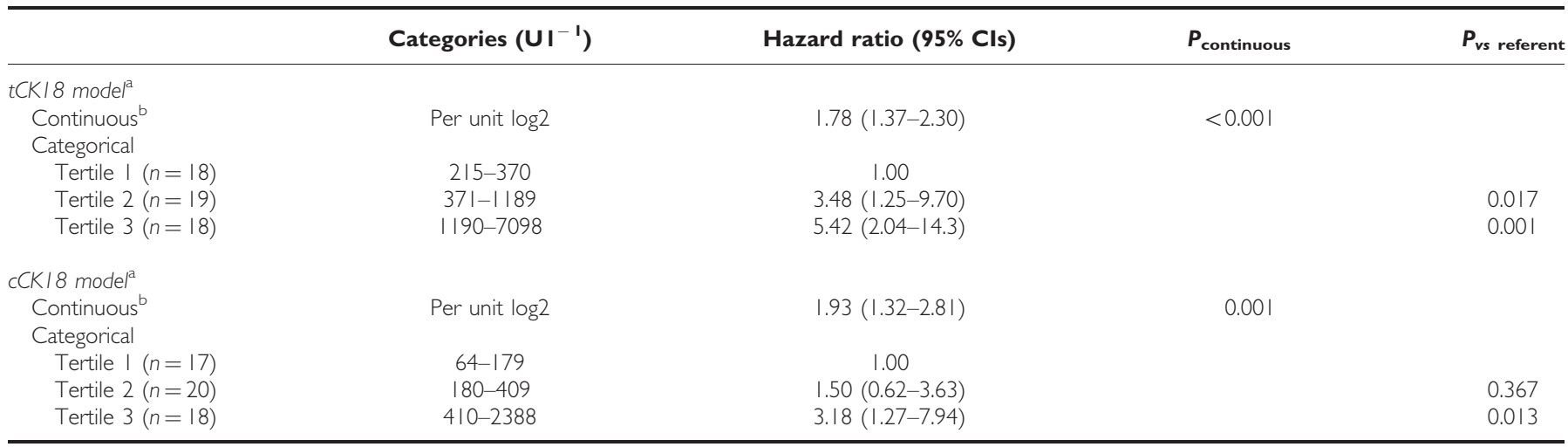

Abbreviation: $\mathrm{Cl}=$ confidence interval. The calibration and performance characteristics for overall survival model for tCK 8 were: Harrell's C-statistic $=0.6949$ and likelihood ratio for goodness-of-fit $=0.867$; for $\mathrm{CCK} 18, \mathrm{C}=0.6384$ and likelihood ratio for goodness-of-fit $=3.489$. ${ }^{\mathrm{a}} \mathrm{All}$ final models included were adjustment for age, sex, stage, and tCK 18

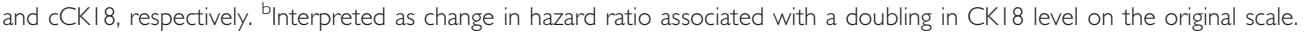

in contrast to patients with clinical benefit from therapy where pre-treatment levels are lower, and after commencement of therapy, either decrease and then flatten (tCK18) or remain constant throughout (cCK18). Collectively, these findings suggest the potential utility of circulating CK18 as a surrogate response biomarker in CRC (Cancer Research UK Glossary, 2011).

\section{Study strengths and limitations}

This study has the advantage that we evaluated previously validated biomarkers where profiles had already been examined in relevant xenograft models (Cummings et al, 2008). We performed prognostic modelling of tCK18- and cCK18-derived data in both surgically treated patients and patients with metastatic disease, and showed different patterns - perhaps, not surprisingly, in patients with metastatic disease where biomarkers levels were most elevated. We then prospectively evaluated changes in serum tCK18 and cCK18 throughout chemotherapy and beyond allowing us to explore longitudinal patterns. This approach will be required to inform future biomarker-adaptive trial designs (Wu et al, 2011). Our study demonstrated the feasibility of undertaking biomarker dynamic studies in 'real-life' clinical practice.

The study findings should be interpreted in light of some limitations. First, patient recruitment was in 'standard of care' settings such that treatment selection and follow-up protocols were not precisely controlled. We addressed these weaknesses with multiple adjustments in our multivariate analyses. Although sample sizes per setting were relatively small, by evaluating a number of clinical scenarios, we were able to test a singular hypothesis; that circulating CK18 is tumour-derived.

\section{Context of other studies}

Elevated levels of circulating tCK18 and cCK18 have been reported in CRC (Koelink et al, 2009; Ausch et al, 2009a, b; Brandt et al, 2010). Similar to the present study, Koelink et al (2009) showed in 49 patients with surgically treated CRC that both biomarkers correlated with stage and tumour volume, but reported significantly independent increased risk of poor outcome in their multivariate analysis for cCK18 but not for tCK18. Ausch et al (2009b) suggested that cCK18 may be a more accurate reflection of tumour burden than tCK18 in patients with surgically resected CRC. Reductions in CK18 levels measured by both tCK18 and cCK18 post surgical resection were noted (Koelink et al, 2009; Ausch et al, 2009b).

The fall in tCK18 seen following chemotherapy in CRC agrees with data previously published in testicular cancer (de Haas et al, 2008) and SCLC (Hou et al, 2009). However, in the former, there 


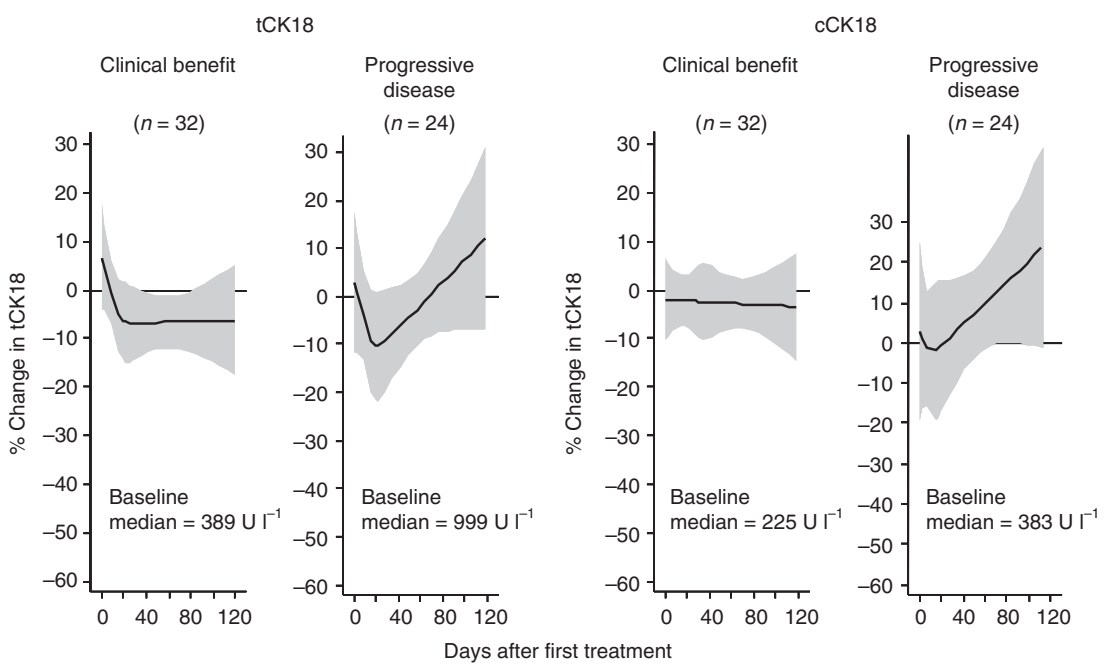

Figure 4 PD monitoring of tCKI8 and CCKI 8 in 56 patients with metastatic CRC undergoing conventional chemotherapy, categorised by treatment response. The y axis is percentage change in biomarker. Curves generated using cubic splines knotted at days 3, I4, and 21 . Shaded area represents $95 \% \mathrm{Cl}$.

were no patients who progressed during chemotherapy; in the latter, samples were taken only throughout the first cycle from patients responding to chemotherapy. Both papers commented that they were unable to distinguish the profile of response from disease progression, as was done in the present study.

Other literature investigating these assays in CRC has not reported on the kinetics of throughout chemotherapy. In the 36 patients with CRC evaluated by Scott et al (2009), they only took samples on day 2. In a mixed study of 35 patients with gastrointestinal cancers (25 with CRC), it was suggested that rising cCK18 levels at the end of the first cycle were associated with clinical benefit (Brandt et al, 2010), and contrasts with data presented here.

\section{Plausible mechanisms and implications}

Initial evaluations of CK18 assays lay in the early assessment of treatment-related tumour death and subsequent prediction of response to therapy (Dean et al, 2009; Gandhi et al, 2011). However, in patients treated with chemotherapy, early CK18 changes may be unhelpful, due to overlap with host toxicity (Greystoke et al, 2011). Although we did not observe any significant changes within the first $48 \mathrm{~h}$, we have shown that for many patients there is a decrease in tCK18, and to a lesser extent cCK18, from 1 week after chemotherapy. We speculate that there is an initial effect of chemotherapy on the population of cells that are chemo-sensitive leading to an initial reduction in overall tumour burden, with the later increases in circulating CK18 reflecting subsequent growth in the population of chemo-resistant cells. If this hypothesis is correct, then these biomarkers could be used as surrogates of treatment response to monitor the development of chemo-resistance and lead to early changes in therapy.

This study highlights the potential utility of these biomarkers to guide care in patients with CRC. In particular, tCK18 might guide drug development in prioritising agents and combinations. On the other hand, cCK18 might have limited utility in monitoring chemotherapy because of the overlap between host toxicity and tumour cell death, but might be most useful in the development of drugs directly targeting the apoptotic pathways (Dean et al, 2011).

\section{Unanswered questions and future research}

Carcinoembryonic antigen is commonly used during chemotherapy and subsequent follow-up in patients with CRC. However, its utility in making early decisions on the effectiveness of therapy is limited by two factors: (i) in approximately a third of patients circulating CEA is undetectable; (ii) in patients with detectable CEA, rising levels can be seen following the first cycle of therapy despite subsequent response (Sorbye and Dahl, 2004). It is likely that the information obtained by measurement of CK18 will be used in conjunction with CEA for clinical decision making, but the optimum method for interpreting these in combination is yet to be determined.

In the future, many MBT agents will be administered in combination with conventional chemotherapy in patients with CRC (Cunningham et al, 2010). Therefore, knowledge of the behaviour of cell death biomarkers following chemotherapy alone will be vital in the interpretation of biomarker profiles in these trials. This study supports the continued deployment of circulating CK18 into biomarker-enhanced trials, particularly in patients undergoing chemotherapy for metastatic CRC.

\section{ACKNOWLEDGEMENTS}

We thank the following funding: AG was funded by the Clinical Pharmacology Fellowship Scheme within the Manchester Cancer Research Centre, Cancer Research UK (C147), and AstraZeneca; ED was funded by a Cancer Research UK Studentship (C237); and the Clinical and Experimental Pharmacology Group at the Paterson Institute was core funded by Cancer Research UK (CD; C147). AGR holds a senior lectureship award supported by the UK Clinical Research Collaboration.

\section{Conflict of interest}

The authors declare no conflict of interest.

\section{Author Contributions}

$\mathrm{AG}, \mathrm{ED}, \mathrm{CD}, \mathrm{AH}, \mathrm{MR}$ and $\mathrm{ARG}$ contributed to study design, analyses and data interpretation. MPS and MR contributed the clinical interpretation in the non-surgically-treated patients. AG and ED contributed equally to the writing of this manuscript. All authors contributed and approved the final manuscript.

Supplementary Information accompanies the paper on British Journal of Cancer website (http://www.nature.com/bjc) 


\section{REFERENCES}

Ausch C, Buxhofer-Ausch V, Olszewski U, Hinterberger W, Ogris E, Schiessel R, Hamilton G (2009a) Caspase-cleaved cytokeratin 18 fragment (M30) as marker of postoperative residual tumor load in colon cancer patients. Eur J Surg Oncol 35(11): 1164-1168

Ausch C, Buxhofer-Ausch V, Olszewski U, Schiessel R, Ogris E, Hinterberger W, Hamilton G (2009b) Circulating cytokeratin 18 fragment m65-a potential marker of malignancy in colorectal cancer patients. J Gastrointest Surg 13(11): 2020-2026

Brandt D, Volkmann X, Anstatt M, Langer F, Manns MP, Schulze-Osthoff K, Bantel H (2010) Serum biomarkers of cell death for monitoring therapy response of gastrointestinal carcinomas. Eur J Cancer 46: 1464-1473

Cancer Research UK Glossary (2011) http://science.cancerresearchuk.org/ funding/find-grant/all-funding-schemes/biomarkers-imaging-discoverydevelopment-project-grants/definitions-glossary/ In Edition (access 2 November 2012)

Cummings J, Hodgkinson C, Odedra R, Sini P, Heaton SP, Mundt KE, Ward TH, Wilkinson RW, Growcott J, Hughes A, Dive C (2008) Preclinical evaluation of M30 and M65 ELISAs as biomarkers of drug induced tumor cell death and antitumor activity. Mol Cancer Ther 7(3): 455-463

Cummings J, Ranson M, Lacasse E, Ganganagari JR, St-Jean M, Jayson G, Durkin J, Dive C (2006) Method validation and preliminary qualification of pharmacodynamic biomarkers employed to evaluate the clinical efficacy of an antisense compound (AEG35156) targeted to the X-linked inhibitor of apoptosis protein XIAP. Br J Cancer 95(1): 42-48

Cummings J, Raynaud F, Jones L, Sugar R, Dive C (2010) Fit-for-purpose biomarker method validation for application in clinical trials of anticancer drugs. Br J Cancer 103(9): 1313-1317

Cummings J, Ward TH, LaCasse E, Lefebvre C, St-Jean M, Durkin J, Ranson M, Dive C (2005) Validation of pharmacodynamic assays to evaluate the clinical efficacy of an antisense compound (AEG 35156) targeted to the X-linked inhibitor of apoptosis protein XIAP. Br J Cancer 92(3): 532-538

Cunningham D, Atkin W, Lenz HJ, Lynch HT, Minsky B, Nordlinger B, Starling N (2010) Colorectal cancer. Lancet 375(9719): 1030-1047

Cuzick J (1985) A Wilcoxon-type test for trend. Stat Med 4(1): 87-90

de Haas EC, di Pietro A, Simpson KL, Meijer C, Suurmeijer AJ, Lancashire LJ, Cummings J, de Jong S, de Vries EG, Dive C, Gietema JA (2008) Clinical evaluation of M30 and M65 ELISA cell death assays as circulating biomarkers in a drug-sensitive tumor, testicular cancer. Neoplasia 10: 1041-1048

Dean E, Jodrell D, Connolly K, Danson S, Jolivet J, Durkin J, Morris S, Jowle D, Ward T, Cummings J, Dickinson G, Aarons L, Lacasse E, Robson L, Dive C, Ranson M (2009) Phase I trial of AEG35156 administered as a 7-day and 3-day continuous intravenous infusion in patients with advanced refractory cancer. J Clin Oncol 27(10): 1660-1666

Dean EJ, Cummings J, Roulston A, Berger M, Ranson M, Blackhall F, Dive C (2011) Optimization of circulating biomarkers of obatoclax-induced cell death in patients with small cell lung cancer. Neoplasia 13: 339-347

Dive C, Smith RA, Garner E, Ward T, George-Smith SS, Campbell F, Greenhalf W, Ghaneh P, Neoptolemos JP (2010) Considerations for the use of plasma cytokeratin 18 as a biomarker in pancreatic cancer. $\mathrm{Br} J$ Cancer 102: 577-582
Gandhi L, Camidge DR, Ribeiro de Oliveira M, Bonomi P, Gandara D, Khaira D, Hann CL, McKeegan EM, Litvinovich E, Hemken PM, Dive C, Enschede SH, Nolan C, Chiu YL, Busman T, Xiong H, Krivoshik AP, Humerickhouse R, Shapiro GI, Rudin CM (2011) Phase I study of navitoclax (ABT-263), a novel bcl-2 family inhibitor, in patients with small-cell lung cancer and other solid tumours. J Clin Oncol 29: 909-916

Greystoke A, Cummings J, Ward T, Simpson K, Renehan A, Butt F, Moore D, Gietema J, Blackhall F, Ranson M, Hughes A, Dive C (2008) Optimisation of circulating biomarkers of cell death for routine clinical use. Ann Oncol 19(5): 990-995

Greystoke A, O’Connor JP, Linton K, Taylor MB, Cummings J, Ward T, Maders F, Hughes A, Ranson M, Illidge TM, Radford J, Dive C (2011) Assessment of circulating biomarkers for potential pharmacodynamic utility in patients with lymphoma. Br J Cancer 104: 719-725

Hou JM, Greystoke A, Lancashire L, Cummings J, Ward T, Board R, Amir E, Hughes S, Krebs M, Hughes A, Ranson M, Lorigan P, Dive C, Blackhall FH (2009) Evaluation of circulating tumor cells and serological cell death biomarkers in small cell lung cancer patients undergoing chemotherapy. Am J Pathol 175: 808-816

Hosmer DW, Lemeshow S (2000) Applied Logistic Regression. Wiley: New York

Koelink PJ, Lamers CB, Hommes DW, Verspaget HW (2009) Circulating cell death products predict clinical outcome of colorectal cancer patients. BMC Cancer 9: 88

McShane LM, Altman DG, Sauerbrei W, Taube SE, Gion M, Clark GM (2005) REporting recommendations for tumour MARKer prognostic studies (REMARK). Br J Cancer 93(4): 387-391

Olofsson MH, Cummings J, Fayad W, Brnjic S, Herrmann R, Berndtsson M, Hodgkinson C, Dean E, Odedra R, Wilkinson RW, Mundt KE, Busk M, Dive C, Linder S (2009) Specific demonstration of drug-induced tumour cell apoptosis in human xenografts models using a plasma biomarker. Cancer Biomark 5(3): 117-125

Renehan AG, Jones J, Potten CS, Shalet SM, O'Dwyer ST (2000) Elevated serum insulin-like growth factor (IGF)-II and IGF binding protein-2 in patients with colorectal cancer. Br J Cancer 83: 1344-1350

Scott LC, Evans TR, Cassidy J, Harden S, Paul J, Ullah R, O’Brien V, Brown $\mathrm{R}$ (2009) Cytokeratin 18 in plasma of patients with gastrointestinal adenocarcinoma as a biomarker of tumour response. $\mathrm{Br} J$ Cancer 101: 410-417

Sorbye H, Dahl O (2004) Transient CEA increase at start of oxaliplatin combination therapy for metastatic colorectal cancer. Acta Oncol 43: 495-498

Steele F (2008) Multilevel models for longitudinal data. J R Stat Soc 171: 5-19

Therasse P, Arbuck SG, Eisenhauer EA, Wanders J, Kaplan RS, Rubinstein L, Verweij J, Van Glabbeke M, van Oosterom AT, Christian MC, Gwyther SG (2000) New guidelines to evaluate the response to treatment in solid tumors. European Organization for Research and Treatment of Cancer, National Cancer Institute of the United States, National Cancer Institute of Canada. J Natl Cancer Inst 92(3): 205-216

Wu W, Shi Q, Sargent DJ (2011) Statistical considerations for the next generation of clinical trials. Semin Oncol 38(4): 598-604

This work is published under the standard license to publish agreement. After 12 months the work will become freely available and the license terms will switch to a Creative Commons Attribution-NonCommercial-Share Alike 3.0 Unported License. 